\title{
Small solutions to semi-linear wave equations with radial data of critical regularity
}

\section{Kunio Hidano}

\begin{abstract}
This paper investigates the problem of global existence of small solutions to semi-linear wave equations with radially symmetric data of critical regularity. Under radial symmetry we focus on the case where the power of nonlinear term is somewhat smaller than the conformal power. Our result covers the case where the power is strictly larger than the John-Glassey exponent in two or three space dimensions. In higher dimension it applies to the equation whose power is strictly larger than the $L^{2}$-critical exponent. The main theorem is therefore an improvement over a previous result due to Lindblad and Sogge. The new ingredient in our proof is an effective use of some weighted estimates of radially symmetric solutions to inhomogeneous wave equations.
\end{abstract}

\section{Introduction and Result}

In this paper we study the Cauchy problem for the semi-linear wave equation

$$
\partial_{t}^{2} u-\Delta u=\lambda|u|^{p-1} u, \quad t \in \mathbb{R}, \quad x \in \mathbb{R}^{n} \quad(p>1, \lambda \in \mathbb{C})
$$

subject to initial data $\left(u(0), \partial_{t} u(0)\right)=(f, g) \in \dot{H}^{s}\left(\mathbb{R}^{n}\right) \times \dot{H}^{s-1}\left(\mathbb{R}^{n}\right)$. Here $\dot{H}^{\sigma}\left(\mathbb{R}^{n}\right)(-n / 2<\sigma<n / 2)$ denotes the homogeneous Sobolev space defined as $\left\{v \in \mathcal{S}^{\prime}\left(\mathbb{R}^{n}\right):\left|D_{x}\right|^{\sigma} v \in L^{2}\left(\mathbb{R}^{n}\right)\right\}$ with the norm $\left\|\left|D_{x}\right|^{\sigma} v\right\|_{L^{2}\left(\mathbb{R}^{n}\right)}$, where we have defined the operator $\left|D_{x}\right|^{\sigma}$ as $\left|D_{x}\right|^{\sigma} v:=\mathcal{F}^{-1}|\xi|^{\sigma} \mathcal{F} v$ using the Fourier transform $\mathcal{F}$ and its inverse transform $\mathcal{F}^{-1}$. Our interest is to study the

2000 Mathematics Subject Classification: Primary: 35L05, 35L15; Secondary: 35L70. Keywords: Wave equation, low-regularity solution, weighted estimate. 
global existence of small $\dot{H}^{s}$-solutions to (1.1). In [11] Lindblad and Sogge studied the $H^{s}$-theory of the equation (1.1) in depth. Assuming

$$
p-1=\frac{4}{n-2 s}, \quad \frac{1}{2} \leq s<\frac{n}{2} \quad(n=2,3), \quad \frac{1}{2} \leq s \leq \frac{3}{2} \quad(n \geq 4)
$$

or, equivalently

$$
\begin{array}{rlrl}
s=\frac{n}{2}-\frac{2}{p-1}, & \frac{4}{n-1} & \leq p-1<\infty(n=2,3), \\
\frac{4}{n-1} & \leq p-1 \leq \frac{4}{n-3}(n \geq 4),
\end{array}
$$

they proved that, for any $(f, g) \in \dot{H}^{s}\left(\mathbb{R}^{n}\right) \times \dot{H}^{s-1}\left(\mathbb{R}^{n}\right)$ with $\left\|\left|D_{x}\right|^{s} f\right\|_{L^{2}\left(\mathbb{R}^{n}\right)}+$ $\left\|\left|D_{x}\right|^{s-1} g\right\|_{L^{2}\left(\mathbb{R}^{n}\right)}$ small, the associated integral equation has a unique global solution (Theorem 2.2 of [11]). From the point of view of the $H^{s}$-theory for nonlinear evolution equations, this result concerns the critical case and is a natural extension of the results due to Pecher (Theorem 2 of [14]), Ginibre and Velo (Proposition 3.3 of [2]) who handled the $H^{1}$-critical case (i.e., $s=1$, $p=1+4 /(n-2))$ for small data. We note that Theorem 2.2 of [11] also covers the case $n \geq 4$ and $3 / 2<s<n / 2$ if $p-1(=4 /(n-2 s))$ is an even integer. Later, their result for higher dimension $n \geq 4$ was improved by Nakamura and Ozawa [13], whose result covers the case of $n \geq 4,3 / 2<s<n / 2$ and $[s]<p-1=4 /(n-2 s)([s]$ denotes the greatest integer not greater than $s \geq 0$ ).

Next let us review the previous results concerning the global existence of small $\dot{H}^{s}$-solutions for $s<1 / 2$. Assuming

$$
p-1=\frac{4}{n+1-4 s}, \quad \frac{n+1}{4 n}<s<\frac{1}{2}
$$

or, equivalently

$$
s=\frac{n+1}{4}-\frac{1}{p-1}, \quad \frac{4 n}{(n-1)(n+1)}<p-1<\frac{4}{n-1}
$$

for general data, or

$$
p-1=\frac{4}{n-2 s}, \quad \frac{1}{2 n}<s<\frac{1}{2}
$$

or, equivalently

$$
s=\frac{n}{2}-\frac{2}{p-1}, \quad \frac{4 n}{(n-1)(n+1)}<p-1<\frac{4}{n-1}
$$


for radially symmetric data, Lindblad and Sogge proved that the associated integral equation has a unique global solution if $\left\|\left|D_{x}\right|^{s} f\right\|_{L^{2}\left(\mathbb{R}^{n}\right)}+$ $\left\|\left|D_{x}\right|^{s-1} g\right\|_{L^{2}\left(\mathbb{R}^{n}\right)}$ is sufficiently small ([12, Theorem 5.9], [11, Theorem 8.1]). In the present paper we intend to show that this result for radially symmetric data holds in a wider range of $s$. Let $K(t)=\left(\sin t\left|D_{x}\right|\right) /\left|D_{x}\right|$ and $\dot{K}(t)=\cos t\left|D_{x}\right|$. We will prove the following

Theorem 1.1. Suppose $n \geq 2, p-1=4 /(n-2 s)$, and

$$
\left\{\begin{aligned}
\frac{1}{4}-\left(\frac{\sqrt{17}}{4}-1\right)<s<\frac{1}{2}, & n=2 \\
\frac{1}{6}-\left(\sqrt{2}-\frac{4}{3}\right)<s<\frac{1}{2}, & n=3 \\
0<s<\frac{1}{2}, & n \geq 4
\end{aligned}\right.
$$

There exists a positive constant $\delta$ depending on $n, p$, and $\lambda$ such that if radially symmetric data $(f, g) \in \dot{H}^{s}\left(\mathbb{R}^{n}\right) \times \dot{H}^{s-1}\left(\mathbb{R}^{n}\right)$ is small so that

$$
\left\|\left|D_{x}\right|^{s} f\right\|_{L^{2}\left(\mathbb{R}^{n}\right)}+\left\|\left|D_{x}\right|^{s-1} g\right\|_{L^{2}\left(\mathbb{R}^{n}\right)} \leq \delta,
$$

then the integral equation

$$
u(t)=\dot{K}(t) f+K(t) g+\lambda \int_{0}^{t} K(t-\tau)|u(\tau)|^{p-1} u(\tau) d \tau
$$

has a unique radially symmetric solution $u \in C\left(\mathbb{R} ; \dot{H}^{s}\left(\mathbb{R}^{n}\right)\right)$ satisfying

$$
\sup _{t \in \mathbb{R}}\left\|\left|D_{x}\right|^{s} u(t)\right\|_{L^{2}\left(\mathbb{R}^{n}\right)}+\left\||x|^{-\alpha_{0}} u\right\|_{L^{q_{0}}\left(\mathbb{R} \times \mathbb{R}^{n}\right)} \leq C \delta
$$

for a suitable constant $C>0$. Here $q_{0}>2$ and $\alpha_{0} \in \mathbb{R}$ are the exponents given (3.2), (3.3) below.

Remark 1.2. Since the way of choosing $q_{0}>2$ is too involved to explain at this stage, we postpone the explanation till Section 3.

The condition in Theorem 1.1 can be restated in terms of $p$, namely, $s=n / 2-2 /(p-1)$ and

$$
\left\{\begin{aligned}
p_{0}(n)-1<p-1<\frac{4}{n-1}, & n=2,3, \\
\frac{4}{n}<p-1<\frac{4}{n-1}, & n \geq 4,
\end{aligned}\right.
$$


where $p_{0}(n)$ is the larger root of the quadratic equation $(n-1) p^{2}-(n+1) p-$ $2=0$, hence $p_{0}(2)=(3+\sqrt{17}) / 2, p_{0}(3)=1+\sqrt{2}, p_{0}(n) \leq 2$ for $n \geq 4$. (It is easily seen that $p_{0}(n)>1+4 / n(n=2,3), p_{0}(4)=2$, and $p_{0}(n)<1+4 / n$ $(n \geq 5)$.) In fact, a result similar to Theorem 1.1 was obtained by Sogge (Theorem 6.1 of Chapter III of [17]) for $n=3$, later by the present author for $n=2,3,4$ [7]. Hence it is for $n \geq 5$ that Theorem 1.1 is essentially new and its proof needs some new ingredients. In this paper we devise how to solve the problem for all $n \geq 2$ at once. To this end, we fully exploit the advantages of some weighted estimates in [7] which have played an essential role in generalizing Theorem 6.1 of Sogge [17] and showing a result similar to Theorem 1.1 for $n=2,3,4$. Though it is elementary, our key observation in the present paper is that the weighted estimate (2.1) below for $e^{i t\left|D_{x}\right|} \varphi$ does hold for $\alpha<0$ as well as $\alpha \geq 0$ under certain condition, whereas in the previous paper [7] the importance of the weighted estimate for $\alpha<0$ was overlooked. Using the Christ-Kiselev lemma [1] along with the standard $T T^{*}$ argument, we derive a useful weighted estimate (see (2.11) below) for the inhomogeneous wave equation from (2.1). Specifically, the estimate (2.11) for $\alpha \tilde{\alpha} \leq 0$ works very well in the contraction-mapping argument, and we will prove our main result for all $n \geq 2$ at once using these weighted estimates.

We remark that the condition $p>p_{0}(n)(n=2,3)$ in Theorem 1.1 is just the same as in the classics of John [9] for $n=3$ and Glassey [4] for $n=2$ concerning the existence of global $C^{2}$-solutions with small, smooth data of compact support. It was also proved by John $(n=3)$ and Glassey $(n=2)$ that there exist finite-time blow-up solutions even for small, smooth data of compact support if $\lambda>0$ and $p<p_{0}(n)$ [9], [3]. (See, e.g., Schaeffer [15] for the blow up for $p=p_{0}(n), n=2,3$.) Interestingly, the number $p_{0}(n)$ plays a prominent role also in the theory of well-posedness for low-regularity, radially symmetric solutions. Indeed, in Chapter III of [17] Sogge proved local-in-time existence of unique solutions for radially symmetric data in $\dot{H}^{1 / 2-1 / p} \times \dot{H}^{-1 / 2-1 / p}$ when $n=3$ and $2<p<p_{0}(3)$. See also [7] where a different proof of this fact was given. ${ }^{1}$ We note that, under the condition $p>1,1 / 2-1 / p>3 / 2-2 /(p-1) \Longleftrightarrow p<p_{0}(3)$. Sogge also showed that the order $1 / 2-1 / p$ is necessary as well as sufficient for local-in-time well-posedness (see page 122 of [17]).

This paper is organized as follows. Section 2 is devoted to the proof of weighted space-time $L^{q}$ estimates for the free wave equation and the inhomogeneous wave equation. In Section 3, using these key estimates and the contraction mapping principle, we prove Theorem 1.1.

\footnotetext{
${ }^{1}$ In [8] it has been proved that the local-in-time existence of unique solutions is true also for $n=2$ and $2<p<p_{0}(2)$ when radially symmetric data are in $\dot{H}^{1 / 2-1 / p} \times \dot{H}^{-1 / 2-1 / p}$. Its proof uses a $(1+2)$-dimensional analogue of the key estimate (6.5) on page 123 of [17].
} 


\section{Weighted space-time $L^{q}$ estimates}

We define $(W \varphi)(t, x)=W(t) \varphi(x)=\mathcal{F}^{-1} e^{i t|\xi|} \mathcal{F} \varphi$. Reviewing the proof in [7] of the weighted $L^{q}$ estimate of $W(t) \varphi$ for radially symmetric data $\varphi$, we begin with showing

Theorem 2.1. Suppose $n \geq 2$. There exists a constant $C>0$ depending on $n, q, \alpha$, and the estimate

$$
\left\||x|^{-\alpha} W \varphi\right\|_{L^{q}\left(\mathbb{R} \times \mathbb{R}^{n}\right)} \leq C\left\|\left|D_{x}\right|^{s} \varphi\right\|_{L^{2}\left(\mathbb{R}^{n}\right)}
$$

holds for radially symmetric data $\varphi \in \dot{H}^{s}\left(\mathbb{R}^{n}\right)$ provided that

$$
\frac{n+1}{q}-\alpha=\frac{n}{2}-s, \quad 2 \leq q<\infty, \quad \frac{n}{q}-\frac{n-1}{2}<\alpha<\frac{n}{q} .
$$

Remark 2.2. In the previous paper [7] the estimate (2.1) was shown under the condition

$$
\frac{n+1}{q}-\alpha=\frac{n}{2}-s, \quad \alpha \geq 0, \quad q \geq 2, \quad \frac{n}{\alpha+\frac{n-1}{2}}<q<\frac{n}{\alpha}
$$

(the last condition $q<n / \alpha$ is interpreted as $q<\infty$ for $\alpha=0$ ), instead of (2.2). It turns out below that we should make much account of the estimate (2.1) also for $\alpha<0$, which, together with the Christ-Kiselev lemma [1], plays a crucial role in the proof of our useful estimate for radially symmetric solutions to inhomogeneous equations (see (2.11)).

Proof . Though its proof is essentially the same as that of Theorem 2.1 of [7], we prove Theorem 2.1 above to make this paper self-contained. Our proof is obviously inspired by that of Proposition 6.6.3 of Sogge [17]. Let $d \sigma$ denote the Lebesgue measure on the unit sphere $S^{n-1} \subset \mathbb{R}^{n}$, and let $\widehat{d \sigma}$ denote the Fourier transform of $d \sigma$, that is,

$$
\widehat{d \sigma}(\xi)=\int_{S_{\omega}^{n-1}} e^{i \omega \cdot \xi} d \sigma \quad(d \sigma=d \sigma(\omega)) .
$$

Since $\varphi$ is radially symmetric and so is $\hat{\varphi}(=\mathcal{F} \varphi)$, we may write $\hat{\varphi}(\xi)$ as $\psi(|\xi|)$. Using the Heaviside function $H(\rho)$, we have

$$
\begin{aligned}
(W \varphi)(t, x) & =(2 \pi)^{-n} \int_{0}^{\infty} e^{i t \rho} \psi(\rho) \rho^{n-1} \widehat{d \sigma}(\rho x) d \rho \\
= & (2 \pi)^{-n+1}\left((2 \pi)^{-1} \int_{\mathbb{R}} e^{i t \rho} H(\rho) \psi(|\rho|) \rho^{n-1} \widehat{d \sigma}(|\rho| x) d \rho\right) .
\end{aligned}
$$


We may view the right-hand side of (2.5) as an inverse Fourier transform in $t$. Using the Sobolev embedding theorem first and then the Plancherel theorem, we have

$$
\begin{aligned}
& \|(W \varphi)(\cdot, x)\|_{L^{q}\left(\mathbb{R}_{t}\right)} \\
& \leq C_{q, n}\left\|\left|D_{t}\right|^{1 / 2-1 / q}\left((2 \pi)^{-1} \int_{\mathbb{R}} e^{i t \rho} H(\rho) \psi(|\rho|) \rho^{n-1} \widehat{d \sigma}(|\rho| x) d \rho\right)\right\|_{L^{2}\left(\mathbb{R}_{t}\right)} \\
& =C\left\|\psi(\rho) \rho^{(1 / 2-1 / q)+n-1} \widehat{d \sigma}(\rho x)\right\|_{L^{2}\left(\mathbb{R}_{\rho}^{+}\right)}
\end{aligned}
$$

for any fixed $x \in \mathbb{R}^{n}$.

To proceed, we recall that $\widehat{d \sigma}$ is a smooth function on $\mathbb{R}^{n}$ satisfying $\widehat{d \sigma}(\xi)=O\left(|\xi|^{-(n-1) / 2}\right.$ ) as $|\xi| \rightarrow \infty$ (see, e.g., Stein [18] on page 348). Using the Minkowski inequality and making the change of variables $y=\rho x$, we obtain

$$
\begin{aligned}
& \left\||x|^{-\alpha} W \varphi\right\|_{L^{q}\left(\mathbb{R} \times \mathbb{R}^{n}\right)} \\
& \leq C\left\|\psi(\rho) \rho^{(1 / 2-1 / q)+n-1}\right\||x|^{-\alpha} \widehat{d \sigma}(\rho x)\left\|_{L^{q}\left(\mathbb{R}_{x}^{n}\right)}\right\|_{L^{2}\left(\mathbb{R}_{\rho}^{+}\right)} \\
& =C\left\|\psi(\rho) \rho^{(1 / 2-1 / q)+\alpha-(n / q)+n-1}\right\||y|^{-\alpha} \widehat{d \sigma}(y)\left\|_{L^{q}\left(\mathbb{R}_{y}^{n}\right)}\right\|_{L^{2}\left(\mathbb{R}_{\rho}^{+}\right)} \\
& \leq C\left\|\psi(\rho) \rho^{(1 / 2-1 / q)+\alpha-(n / q)+n-1}\right\|_{L^{2}\left(\mathbb{R}_{\rho}^{+}\right)} \\
& =C\left\||\xi|^{(1 / 2-1 / q)+\alpha-(n / q)+(n-1) / 2} \hat{\varphi}\right\|_{L^{2}\left(\mathbb{R}^{n}\right)} \\
& =C\left\|\left|D_{x}\right|^{(n / 2)-((n+1) / q)+\alpha} \varphi\right\|_{L^{2}\left(\mathbb{R}^{n}\right)} .
\end{aligned}
$$

Here we have handled the $L^{q}\left(\mathbb{R}_{y}^{n}\right)$-norm of $|y|^{-\alpha} \widehat{d \sigma}(y)$ as

$$
\left\||y|^{-\alpha} \widehat{d \sigma}(y)\right\|_{L^{q}\left(\left\{y \in \mathbb{R}^{n}:|y|<1\right\}\right)}+\left\||y|^{-\alpha} \widehat{d \sigma}(y)\right\|_{L^{q}\left(\left\{y \in \mathbb{R}^{n}:|y|>1\right\}\right)} \leq C,
$$

using the decay property of $\widehat{d \sigma}$ and the last condition in (2.2). We have finished the proof.

In what follows we denote by $q^{\prime}$ the Hölder conjugate exponent of $q$ : $1 / q+1 / q^{\prime}=1$. By virtue of the Christ-Kiselev lemma (see Theorem 1.2 of [1], see also Smith and Sogge [16], Tao [20]) we can derive the following key estimates for radially symmetric solutions to inhomogeneous equations directly from the estimate $(2.1)$ for $W(t) \varphi$. 
Theorem 2.3. Suppose $n \geq 2,2 \leq q<\infty, 2 \leq \tilde{q}<\infty,(q, \tilde{q}) \neq(2,2)$, and

$$
\frac{n}{q}-\frac{n-1}{2}<\alpha<\frac{n}{q}, \quad \frac{n}{\tilde{q}}-\frac{n-1}{2}<\tilde{\alpha}<\frac{n}{\tilde{q}} .
$$

Set

$$
s:=\frac{n}{2}+\alpha-\frac{n+1}{q}, \quad \tilde{s}:=\frac{n}{2}+\tilde{\alpha}-\frac{n+1}{\tilde{q}} .
$$

The estimate

$$
\left\||x|^{-\alpha} \int_{0}^{t} W(t-\tau) F(\tau) d \tau\right\|_{L^{q}\left(\mathbb{R} \times \mathbb{R}^{n}\right)} \leq C\left\||x|^{\tilde{\alpha}}\left|D_{x}\right|^{s+\tilde{s}} F\right\|_{L^{\tilde{q}^{\prime}}\left(\mathbb{R} \times \mathbb{R}^{n}\right)}
$$

holds for radially symmetric (in $x$ ) $F$.

Theorem 2.4. Suppose $n \geq 2,2 \leq \hat{q}<\infty$, and

$$
\frac{n}{\hat{q}}-\frac{n-1}{2}<\hat{\alpha}<\frac{n}{\hat{q}} \text {. }
$$

Set

$$
\hat{s}:=\frac{n}{2}+\hat{\alpha}-\frac{n+1}{\hat{q}} .
$$

The estimate

$$
\left\|\left|D_{x}\right|^{-\hat{s}} \int_{0}^{t} W(t-\tau) F(\tau) d \tau\right\|_{L^{\infty}\left(\mathbb{R} ; L^{2}\left(\mathbb{R}^{n}\right)\right)} \leq C\left\||x|^{\hat{\alpha}} F\right\|_{L^{\hat{q}^{\prime}\left(\mathbb{R} \times \mathbb{R}^{n}\right)}}
$$

holds for radially symmetric (in $x) F$.

Corollary 2.5. Suppose $n \geq 2,2 \leq q<\infty, 2 \leq \tilde{q}<\infty,(q, \tilde{q}) \neq(2,2)$,

$$
\frac{n}{q}-\frac{n-1}{2}<\alpha<\frac{n}{q}, \frac{n}{\tilde{q}}-\frac{n-1}{2}<\tilde{\alpha}<\frac{n}{\tilde{q}},
$$

and

$$
\frac{n}{2}+\alpha-\frac{n+1}{q}+\frac{n}{2}+\tilde{\alpha}-\frac{n+1}{\tilde{q}}=1 .
$$

The estimate

$$
\begin{aligned}
& \left\|\left|D_{x}\right|^{1-(n / 2)-\tilde{\alpha}+(n+1) / \tilde{q}} \int_{0}^{t} K(t-\tau) F(\tau) d \tau\right\|_{L^{\infty}\left(\mathbb{R} ; L^{2}\left(\mathbb{R}^{n}\right)\right)} \\
& +\left\||x|^{-\alpha} \int_{0}^{t} K(t-\tau) F(\tau) d \tau\right\|_{L^{q}\left(\mathbb{R} \times \mathbb{R}^{n}\right)} \leq C\left\||x|^{\tilde{\alpha}} F\right\|_{L^{\tilde{q}}\left(\mathbb{R} \times \mathbb{R}^{n}\right)}
\end{aligned}
$$

holds for radially symmetric (in $x) F$. 
Proof of Theorem 2.3. As is well-known, we have

$$
W^{*} F=\int_{\mathbb{R}} W(-\tau) F(\tau) d \tau
$$

Theorem 2.1 therefore yields

$$
\left\|\left|D_{x}\right|^{-\tilde{s}} W^{*} F\right\|_{L^{2}\left(\mathbb{R}^{n}\right)} \leq C\left\||x|^{\tilde{\alpha}} F\right\|_{L^{\tilde{q}^{\prime}\left(\mathbb{R} \times \mathbb{R}^{n}\right)}}
$$

and

$$
\begin{aligned}
& \left\||x|^{-\alpha} \int_{\mathbb{R}} W(t-\tau) F(\tau) d \tau\right\|_{L^{q}\left(\mathbb{R} \times \mathbb{R}^{n}\right)}=\left\||x|^{-\alpha} W W^{*} F\right\|_{L^{q}\left(\mathbb{R} \times \mathbb{R}^{n}\right)} \\
& \leq C\left\|\left|D_{x}\right|^{s} W^{*} F\right\|_{L^{2}\left(\mathbb{R}^{n}\right)}=C\left\|\left|D_{x}\right|^{-\tilde{s}} W^{*}\left|D_{x}\right|^{s+\tilde{s}} F\right\|_{L^{2}\left(\mathbb{R}^{n}\right)} \\
& \leq C\left\||x|^{\tilde{\alpha}}\left|D_{x}\right|^{s+\tilde{s}} F\right\|_{L^{\tilde{q}^{\prime}}\left(\mathbb{R} \times \mathbb{R}^{n}\right)} .
\end{aligned}
$$

Taking account of the assumption $(q, \tilde{q}) \neq(2,2)$, we may apply the ChristKiselev lemma [1] and we obtain the estimate (2.9). The proof of Theorem 2.3 has been finished.

Proof of Theorem 2.4. By the conservation law and (2.13) we have for any fixed $t \in \mathbb{R}$

$$
\begin{aligned}
& \left\|\left|D_{x}\right|^{-\hat{s}} W(t) W^{*} F\right\|_{L^{2}\left(\mathbb{R}^{n}\right)}=\left\|W(t)\left|D_{x}\right|^{-\hat{s}} W^{*} F\right\|_{L^{2}\left(\mathbb{R}^{n}\right)} \\
& =\left\|\left|D_{x}\right|^{-\hat{s}} W^{*} F\right\|_{L^{2}\left(\mathbb{R}^{n}\right)} \leq C\left\||x|^{\hat{\alpha}} F\right\|_{L^{\hat{q}^{\prime}}\left(\mathbb{R} \times \mathbb{R}^{n}\right)},
\end{aligned}
$$

which leads to

$$
\left\|\left|D_{x}\right|^{-\hat{s}} W(t) W^{*} F\right\|_{L^{\infty}\left(\mathbb{R} ; L^{2}\left(\mathbb{R}^{n}\right)\right)} \leq C\left|\left\||x|^{\hat{\alpha}} F\right\|_{L^{\hat{q}^{\prime}\left(\mathbb{R} \times \mathbb{R}^{n}\right)}} .\right.
$$

Again we can use the Christ-Kiselev lemma to derive the estimate (2.10) directly from (2.15). The proof of Theorem 2.4 has been finished.

Proof of Corollary 2.5. Since

$$
K(t)=\frac{\sin t\left|D_{x}\right|}{\left|D_{x}\right|}=\frac{e^{i t\left|D_{x}\right|}-e^{-i t\left|D_{x}\right|}}{2 i\left|D_{x}\right|}
$$

and all the results in Theorems 2.1, 2.3 and 2.4 are obviously valid also for $e^{-i t\left|D_{x}\right|}$, the estimate $(2.11)$ is an immediate consequence of Theorems 2.3 and 2.4 . 
Remark 2.5. We make three remarks on the estimate (2.11). In the previous paper [7] the author showed as an immediate consequence of (2.1)

$$
\begin{aligned}
& \left\|\left|D_{x}\right|^{s} \int_{0}^{t} K(t-\tau) F(\tau) d \tau\right\|_{L^{\infty}\left(\mathbb{R} ; L^{2}\left(\mathbb{R}^{n}\right)\right)} \\
& \quad+\left\||x|^{-\alpha} \int_{0}^{t} K(t-\tau) F(\tau) d \tau\right\|_{L_{\left(\mathbb{R} \times \mathbb{R}^{n}\right)}} \\
& \leq C \int_{\mathbb{R}}\left\|\left|D_{x}\right|^{-1+s} F(\tau)\right\|_{L^{2}\left(\mathbb{R}^{n}\right)} d \tau
\end{aligned}
$$

for $n \geq 2$ and $q, s, \alpha$ satisfying (2.3). Combined with the inequality

$$
\begin{aligned}
& \left\|\left|D_{x}\right|^{-\tilde{s}} v\right\|_{L^{2}\left(\mathbb{R}^{n}\right)} \\
& \leq C\left\|r^{-(n / 2)+\tilde{s}}\right\| v(r \cdot)\left\|_{L^{2}\left(S^{n-1}\right)}\right\|_{L^{1}\left((0, \infty) ; r^{n-1} d r\right)}, \quad \frac{1}{2}<\tilde{s}<\frac{n}{2}
\end{aligned}
$$

which is a dual version of the trace lemma (see e.g., Li and Zhou [10], Hidano [6])

$$
\sup _{r>0} r^{(n / 2)-\tilde{s}}\|v(r \cdot)\|_{L^{2}\left(S^{n-1}\right)} \leq C\left\|\left|D_{x}\right|^{\tilde{s}} v\right\|_{L^{2}\left(\mathbb{R}^{n}\right)}, \quad \frac{1}{2}<\tilde{s}<\frac{n}{2},
$$

the inequality (2.16) leads to

$$
\begin{aligned}
& \left\|\left|D_{x}\right|^{s} \int_{0}^{t} K(t-\tau) F(\tau) d \tau\right\|_{L^{\infty}\left(\mathbb{R} ; L^{2}\left(\mathbb{R}^{n}\right)\right)} \\
& +\left\||x|^{-\alpha} \int_{0}^{t} K(t-\tau) F(\tau) d \tau\right\|_{L^{q}\left(\mathbb{R} \times \mathbb{R}^{n}\right)} \leq C\left\||x|^{-(n / 2)+(1-s)} F\right\|_{L^{1}\left(\mathbb{R} \times \mathbb{R}^{n}\right)}
\end{aligned}
$$

if $1 / 2<1-s<n / 2$, in addition. Actually, the estimates (2.16) obviously holds for $n \geq 2$ and $q, s, \alpha$ satisfying (2.2). Since $s=n / 2-(n+1) / q+\alpha$ (see (2.2)), the condition $1 / 2<1-s<n / 2$ in (2.18) is equivalent to $-(n-1) / 2<-(n / 2)+(1-s)<0$. Hence we find that the estimate $(2.11)$ is valid also in the limiting case $\tilde{q}=\infty$ for radially symmetric (in $x$ ) $F$.

The second remark on the estimate $(2.11)$ is made for the case $(q, \tilde{q})=$ $(2,2)$. In the framework of the $L^{2}$ theory Sugimoto established some weighted estimates for the wave equation. It follows from Corollary 2.6 of [19] that

$$
\left\||x|^{-\alpha} \int_{0}^{t} K(t-\tau) F(\tau) d \tau\right\|_{L^{2}\left(\mathbb{R} \times \mathbb{R}^{n}\right)} \leq C\left\||x|^{\tilde{\alpha}} F\right\|_{L^{2}\left(\mathbb{R} \times \mathbb{R}^{n}\right)}
$$

if $1 / 2<\alpha<n / 2,1 / 2<\tilde{\alpha}<n / 2, \alpha+\tilde{\alpha}=2$. Indeed, we obtain this inequality (2.19) just by taking $\sigma=\eta=0$ and $s+\alpha=0$ in Corollary 2.6 
of [19]. Actually, Sugimoto studied the problem without the radial symmetry of $F$ and the $L^{2}$-estimate he proved is much more general in the sense that it states the property of regularity in the angular variables. Since the radial estimate (2.10) is true for $\hat{q}=2$, we conclude that the estimate (2.11) in fact holds also for $(q, \tilde{q})=(2,2)$ when $F$ is radially symmetric in $x$.

The final remark is made on the relation between our estimate (2.11) and the Harmse estimate used in the proof of Theorem 8.1 of [11]. In [5] Harmse proved the estimate

$$
\begin{aligned}
& \left\|\int_{0}^{t} K(t-\tau) F(\tau) d \tau\right\|_{L^{q}\left(\mathbb{R} \times \mathbb{R}^{n}\right)} \leq C\|F\|_{L^{\tilde{q}^{\prime}}\left(\mathbb{R} \times \mathbb{R}^{n}\right)} \\
& \frac{n+1}{2 n}-\frac{2}{n+1}<\frac{1}{q}<\frac{n-1}{2 n}, \frac{n+1}{q}+2=\frac{n+1}{\tilde{q}^{\prime}}
\end{aligned}
$$

without the assumption of radial symmetry of $F$. See Theorem 2.3 of [5]. As far as radially symmetric solutions are concerned, we can derive (2.20) directly from (2.11) by taking $\alpha=\tilde{\alpha}=0$. Thus our estimate (2.11) may be regarded as a weighted version of the Harmse estimate (2.20).

\section{Proof of Theorem 1.1}

We recall that $p_{0}(n)$ is the larger root of the equation $(n-1) p^{2}-(n+1) p-2=$ 0 , and it satisfies $p_{0}(n)>1+(4 / n)(n=2,3), p_{0}(4)=2, p_{0}(n)<1+(4 / n)$ $(n \geq 5)$. We start with the following observation.

Proposition 3.1. Suppose $n \geq 2$. The inequality

$$
\max \left\{\frac{1}{2 p}, \frac{2}{p-1}-\frac{n-1}{2}, \frac{2}{p-1}-\frac{n}{p}\right\}<\frac{1}{p}
$$

holds for any $p$ with $\max \left\{p_{0}(n), 1+(4 / n)\right\}<p<1+4 /(n-1)$.

Proof. We note

$$
\frac{2}{p-1}-\frac{n-1}{2}>\frac{2}{p-1}-\frac{n}{p} \Longleftrightarrow p<\frac{2 n}{n-1} .
$$

Since

$$
1+\frac{4}{n-1} \leq \frac{2 n}{n-1} \Longleftrightarrow n \geq 3
$$

we see

$$
\frac{2}{p-1}-\frac{n-1}{2}>\frac{2}{p-1}-\frac{n}{p}
$$


for $n \geq 3$, and

$$
\begin{aligned}
& \frac{2}{p-1}-\frac{n-1}{2} \geq \frac{2}{p-1}-\frac{n}{p} \quad \text { if } p_{0}(2)<p \leq 4, \\
& \frac{2}{p-1}-\frac{n-1}{2} \leq \frac{2}{p-1}-\frac{n}{p} \text { if } 4 \leq p<5
\end{aligned}
$$

for $n=2$. As is easily observed, we have the equivalence

$$
\frac{2}{p-1}-\frac{n-1}{2}<\frac{1}{p} \Longleftrightarrow(n-1) p^{2}-(n+1) p-2>0,
$$

which shows Proposition 3.1 for $n \geq 3$ and for $n=2, p_{0}(2)<p \leq 4$.

It remains to consider the case $n=2,4<p<5$. What we need to verify is the inequality

$$
\frac{2}{p-1}-\frac{2}{p}<\frac{1}{p} \quad(4<p<5)
$$

which is obviously true. We have finished the proof of Proposition 3.1.

We note that the inequality $p>1+(4 / n)$ is equivalent to $2 /(p-1)-$ $(n-1) / 2<1 / 2$, and that the inequality $2 /(p-1)-n / p<1 / 2$ holds when $n=2,4<p<5$. In view of Proposition 3.1 we can choose $q_{0}>2$ satisfying

$$
\max \left\{\frac{1}{2 p}, \frac{2}{p-1}-\frac{n-1}{2}, \frac{2}{p-1}-\frac{n}{p}\right\}<\frac{1}{q_{0}}<\frac{1}{p}
$$

for any $n \geq 2$ and any $p$ with $\max \left\{p_{0}(n), 1+(4 / n)\right\}<p<1+4 /(n-1)$. For this exponent $q_{0}$ we set

$$
\alpha_{0}=\frac{n+1}{q_{0}}-\frac{2}{p-1} .
$$

Employing these two exponents $q_{0}$ and $\alpha_{0}$, we define the space $X$ of functions on $\mathbb{R}^{n}$ as follows:

(3.4) $X:=\{v=v(t, x) \mid v$ is radially symmetric in $x$ and

$$
\begin{aligned}
& v \in C\left(\mathbb{R} ; \dot{H}^{s_{0}}\left(\mathbb{R}^{n}\right)\right), \\
& \left.\sup _{t \in \mathbb{R}}\left\|\left|D_{x}\right|^{s_{0}} v(t, \cdot)\right\|_{L^{2}\left(\mathbb{R}^{n}\right)}+\left\||x|^{-\alpha_{0}} v\right\|_{L^{q_{0}\left(\mathbb{R} \times \mathbb{R}^{n}\right)}}<\infty\right\} .
\end{aligned}
$$

Here and in what follows, we set $s_{0}:=n / 2-2 /(p-1)$. Choosing $q_{1}$ and $\alpha_{1}$ in such a way that they satisfy

$$
p q_{1}^{\prime}=q_{0}, \quad-\frac{\alpha_{1}}{p}=\alpha_{0}
$$

and using the key estimate (2.11) with $\tilde{q}=q_{1}, \tilde{\alpha}=\alpha_{1}$, we carry out the contraction-mapping argument. 
To proceed, we must make sure of the following conditions: $2 \leq q_{1}<\infty$,

$$
\frac{n}{q_{j}}-\frac{n-1}{2}<\alpha_{j}<\frac{n}{q_{j}} \quad(j=0,1)
$$

and

$$
\frac{n}{2}+\alpha_{0}-\frac{n+1}{q_{0}}+\frac{n}{2}+\alpha_{1}-\frac{n+1}{q_{1}}=1 .
$$

Let us start with the verification of $2 \leq q_{1}<\infty$. By the definition of $q_{1}$ (see $(3.5)$ ) we see $0<1 / q_{1} \leq 1 / 2 \Longleftrightarrow 1 /(2 p) \leq 1 / q_{0}<1 / p$, and the last condition is indeed satisfied (see (3.2)).

We next verify (3.6) for $j=0$. By the definition of $\alpha_{0}$ (see (3.3)) we find that the condition (3.6) for $j=0$ is equivalent to

$$
\frac{2}{p-1}-\frac{n-1}{2}<\frac{1}{q_{0}}<\frac{2}{p-1} .
$$

Because of $1 / p<2 /(p-1)$ we find that the condition (3.6) is surely satisfied for $j=0$ (see (3.2)). In order to verify (3.6) for $j=1$ we recall the definition of $\alpha_{0}, q_{1}$ and $\alpha_{1}$ (see (3.3), (3.5)). It is seen that the condition (3.6) for $j=1$ is equivalent to

$$
\frac{2}{p-1}-\frac{n}{p}<\frac{1}{q_{0}}<\frac{2}{p-1}-\frac{n+1}{2 p}
$$

Since

$$
\frac{1}{p}<\frac{2}{p-1}-\frac{n+1}{2 p} \Longleftrightarrow p<1+\frac{4}{n-1},
$$

we find that the condition (3.6) is satisfied also for $j=1$ (see (3.2)).

Finally, the exponent $\alpha_{0}$ has in fact been defined in (3.3) so that the condition (3.7) is satisfied. Hence we have finished the verification of the conditions in Corollary 2.4.

We are ready to solve the integral equation (1.6) by using the estimates (2.1) and (2.11). In what follows we always assume $n \geq 2$ and $\max \left\{p_{0}(n), 1+(4 / n)\right\}<p<1+4 /(n-1)$. An application of $(2.1)$ shows

Proposition 3.2. Suppose $f \in \dot{H}^{s_{0}}\left(\mathbb{R}^{n}\right), g \in \dot{H}^{s_{0}-1}\left(\mathbb{R}^{n}\right)$. If $f$ and $g$ are radially symmetric, then $\dot{K}(t) f, K(t) g \in X$ and the estimate

$$
\begin{aligned}
& \sup _{t \in \mathbb{R}}\left\|\left|D_{x}\right|^{s_{0}} u_{0}(t)\right\|_{L^{2}\left(\mathbb{R}^{n}\right)}+|||x|^{-\alpha_{0}} u_{0} \|_{L^{q_{0}\left(\mathbb{R} \times \mathbb{R}^{n}\right)}} \\
& \leq C\left(\left\|\left|D_{x}\right|^{s_{0}} f\right\|_{L^{2}\left(\mathbb{R}^{n}\right)}+\left\|\left|D_{x}\right|^{s_{0}-1} g\right\|_{L^{2}\left(\mathbb{R}^{n}\right)}\right)
\end{aligned}
$$

holds for a suitable constant $C>0$ independent of $f, g$. Here $u_{0}$ denotes $\dot{K}(t) f+K(t) g$. 
Proof. Obviously, $\dot{K}(t) f, K(t) g \in C\left(\mathbb{R} ; \dot{H}^{s_{0}}\left(\mathbb{R}^{n}\right)\right)$, and they are radially symmetric. We note that

$$
\dot{K}(t)=\frac{e^{i t\left|D_{x}\right|}+e^{-i t\left|D_{x}\right|}}{2}, \quad K(t)=\frac{e^{i t\left|D_{x}\right|}-e^{-i t\left|D_{x}\right|}}{2 i\left|D_{x}\right|} .
$$

We also note that the result of Theorem 2.1 is valid also for $e^{-i t\left|D_{x}\right|}$. Since the condition (2.2) is satisfied by $q_{0}, s_{0}$ and $\alpha_{0}$, the estimate (3.8) is an immediate consequence of Theorem 2.1 and the energy conservation.

Proposition 3.3. For $v \in X$

$$
\int_{0}^{t} K(t-\tau)|v(\tau)|^{p-1} v(\tau) d \tau \in X
$$

Moreover, the estimate

$$
\begin{aligned}
& \sup _{t \in \mathbb{R}}\left\|\left|D_{x}\right|^{s_{0}} \int_{0}^{t} K(t-\tau)\left(\left|v_{1}(\tau)\right|^{p-1} v_{1}(\tau)-\left|v_{2}(\tau)\right|^{p-1} v_{2}(\tau)\right) d \tau\right\|_{L^{2}\left(\mathbb{R}^{n}\right)} \\
& +\left\||x|^{-\alpha_{0}} \int_{0}^{t} K(t-\tau)\left(\left|v_{1}(\tau)\right|^{p-1} v_{1}(\tau)-\left|v_{2}(\tau)\right|^{p-1} v_{2}(\tau)\right) d \tau\right\|_{L^{q_{0}\left(\mathbb{R} \times \mathbb{R}^{n}\right)}} \\
& \leq C\left(\sum_{j=1}^{2}\left\||x|^{-\alpha_{0}} v_{j}\right\|_{\left.L^{q_{0}\left(\mathbb{R} \times \mathbb{R}^{n}\right)}\right)}\right)^{p-1}\left\||x|^{-\alpha_{0}}\left(v_{1}-v_{2}\right)\right\|_{L^{q_{0}\left(\mathbb{R} \times \mathbb{R}^{n}\right)}}
\end{aligned}
$$

holds for all $v_{1}, v_{2} \in X$.

Proof. First of all, we recall the definition (3.5) of two exponents $q_{1}$ and $\alpha_{1}$. For the purpose of showing Proposition 3.3 we need to verify the fact

$$
\left|D_{x}\right|^{s_{0}} \int_{0}^{t} K(t-\tau) F(\tau) d \tau \in\left(C \cap L^{\infty}\right)\left(\mathbb{R} ; L^{2}\left(\mathbb{R}^{n}\right)\right)
$$

for radially symmetric (in $x) F$ satisfying $|x|^{\alpha_{1}} F \in L^{q_{1}^{\prime}}\left(\mathbb{R} \times \mathbb{R}^{n}\right)$. To make sure of the continuity in the time variable, we choose a sequence of radially symmetric (in $x$ ) functions $\left\{G_{j}\right\}_{j \in \mathbb{N}} \subset C_{0}^{\infty}\left(\mathbb{R} \times \mathbb{R}^{n}\right)$ such that $G_{j} \rightarrow|x|^{\alpha_{1}} F$ in $L^{q_{1}^{\prime}}\left(\mathbb{R} \times \mathbb{R}^{n}\right)(j \rightarrow \infty)$ by the density of $C_{0}^{\infty}\left(\mathbb{R} \times \mathbb{R}^{n}\right)$ in $L^{q_{1}^{\prime}}\left(\mathbb{R} \times \mathbb{R}^{n}\right)$. Setting $F_{j}:=|x|^{-\alpha_{1}} G_{j}$, we claim that $\left|D_{x}\right|^{-1+s_{0}} F_{j} \in L_{\text {loc }}^{1}\left(\mathbb{R} ; L^{2}\left(\mathbb{R}^{n}\right)\right)$. Indeed, by the Sobolev embedding theorem, we know $L^{l}\left(\mathbb{R}^{n}\right) \subset \dot{H}^{-\left(1-s_{0}\right)}\left(\mathbb{R}^{n}\right)(1 / l=$ $\left.1 / 2+\left(1-s_{0}\right) / n\right)$. It therefore suffices to show $|x|^{-\alpha_{1}} \in L^{l}(B)(B:=\{x \in$ $\left.\mathbb{R}^{n}:|x|<1\right\}$ ) for the verification of the claim. We see

$$
\begin{aligned}
|x|^{-\alpha_{1}} \in L^{l}(B) & \Longleftrightarrow \alpha_{1}<\frac{n}{l} \Longleftrightarrow \alpha_{1}<\frac{n}{2}+1-s_{0} \\
& \Longleftrightarrow \alpha_{1}<\frac{n}{2}+1-\left(\frac{n}{2}-\frac{n+1}{q_{0}}+\alpha_{0}\right) .
\end{aligned}
$$


Here the last equivalence is due to $s_{0}=(n / 2)-(n+1) / q_{0}+\alpha_{0}$. Moreover, using (3.7), we see that

$$
\begin{aligned}
\left(\frac{n}{2}\right. & \left.-\frac{n+1}{q_{0}}+\alpha_{0}\right)+\alpha_{1}<\frac{n}{2}+1 \\
& \Longleftrightarrow\left(1-\frac{n}{2}-\alpha_{1}+\frac{n+1}{q_{1}}\right)+\alpha_{1}<\frac{n}{2}+1 \Longleftrightarrow \frac{n+1}{q_{1}}<n .
\end{aligned}
$$

Since $1 / q_{1}=1-\left(p / q_{0}\right)$ (see $\left.(3.5)\right)$, the last inequality may be rewritten as

$$
\frac{1}{p(n+1)}<\frac{1}{q_{0}}
$$

Because of $1 /(2 p)<1 / q_{0}$ (see (3.2)) the condition (3.12) is obviously satisfied, and our claim has been verified.

By virtue of our claim $\left|D_{x}\right|^{-1+s_{0}} F_{j} \in L_{\text {loc }}^{1}\left(\mathbb{R} ; L^{2}\left(\mathbb{R}^{n}\right)\right)$ we find by the standard argument

$$
\begin{aligned}
\left|D_{x}\right|^{s_{0}} & \int_{0}^{t} K(t-\tau) F_{j}(\tau) d \tau \\
& =\int_{0}^{t}\left(\sin (t-\tau)\left|D_{x}\right|\right)\left|D_{x}\right|^{-1+s_{0}} F_{j}(\tau) d \tau \in C\left(\mathbb{R} ; L^{2}\left(\mathbb{R}^{n}\right)\right)
\end{aligned}
$$

$(j=1,2, \ldots)$, and by the estimate $(2.11)$

$$
\begin{aligned}
\sup _{t \in \mathbb{R}} & \left\|\left|D_{x}\right|^{s_{0}} \int_{0}^{t} K(t-\tau)\left(F_{j}(\tau)-F_{k}(\tau)\right) d \tau\right\|_{L^{2}\left(\mathbb{R}^{n}\right)} \\
& \leq C\left\||x|^{\alpha_{1}}\left(F_{j}-F_{k}\right)\right\|_{L^{q_{1}^{\prime}\left(\mathbb{R} \times \mathbb{R}^{n}\right)}}=C\left\|G_{j}-G_{k}\right\|_{L^{q_{1}^{\prime}\left(\mathbb{R} \times \mathbb{R}^{n}\right)}} \rightarrow 0
\end{aligned}
$$

as $j, k \rightarrow \infty$. Due to the completeness of $\left(C \cap L^{\infty}\right)\left(\mathbb{R} ; L^{2}\left(\mathbb{R}^{n}\right)\right)$ the limit function

$$
\left|D_{x}\right|^{s_{0}} \int_{0}^{t} K(t-\tau) F(\tau) d \tau
$$

is also in $\left(C \cap L^{\infty}\right)\left(\mathbb{R} ; L^{2}\left(\mathbb{R}^{n}\right)\right)$, which shows (3.11).

Now we are ready to complete the proof of Proposition 3.3. The radial symmetry of the integral in (3.9) is obvious. The exponents $q_{1}$ and $\alpha_{1}$ have been chosen in (3.5) so that the property (3.9) is a direct consequence of (2.11) and (3.11). Using the basic inequality

$$
\left.|| v_{1}\right|^{p-1} v_{1}-\left|v_{2}\right|^{p-1} v_{2}\left|\leq C\left(\left|v_{1}\right|+\left|v_{2}\right|\right)^{p-1}\right| v_{1}-v_{2} \mid,
$$

we can obtain the estimate (3.10) in quite the same way. The proof of Proposition 3.3 has been finished. 
We are in a position to complete the proof of Theorem 1.1. For $M>0$ let us define

$$
X_{M}:=\left\{v \in X\left|\sup _{t \in \mathbb{R}}\left\|\left|D_{x}\right|^{s_{0}} v(t, \cdot)\right\|_{L^{2}\left(\mathbb{R}^{n}\right)}+\left\||x|^{-\alpha_{0}} v\right\|_{L^{q_{0}\left(\mathbb{R} \times \mathbb{R}^{n}\right)}} \leq M\right\} .\right.
$$

Endowed with the metric

$$
d(v, w):=\sup _{t \in \mathbb{R}}\left\|\left|D_{x}\right|^{s_{0}}(v(t, \cdot)-w(t, \cdot))\right\|_{L^{2}\left(\mathbb{R}^{n}\right)}+\left\||x|^{-\alpha_{0}}(v-w)\right\|_{L^{q_{0}}\left(\mathbb{R} \times \mathbb{R}^{n}\right)},
$$

the set $X_{M}$ is a complete metric space. Defining the operator

$$
N(v):=\dot{K}(t) f+K(t) g+\lambda \int_{0}^{t} K(t-\tau)|v(\tau)|^{p-1} v(\tau) d \tau,
$$

we find by Propositions 3.2 and 3.3 that there exists a constant $\delta>0$ depending on $n, p$, and $\lambda$ such that if $\left\|\left|D_{x}\right|^{s_{0}} f\right\|_{L^{2}\left(\mathbb{R}^{n}\right)}+\left\|\left|D_{x}\right|^{s_{0}-1} g\right\|_{L^{2}\left(\mathbb{R}^{n}\right)} \leq \delta$, then the operator $N$ has a unique fixed point in $X_{C \delta}$. Here $C$ is a suitable positive constant, and the fixed point is a solution to the integral equation (1.6) which is unique in $X_{C \delta}$. We have finished the proof of Theorem 1.1.

\section{References}

[1] Christ, M. And Kiselev, A.: Maximal functions associated to filtrations. J. Funct. Anal. 179 (2001), 409-425.

[2] Ginibre, J. And Velo, G.: Scattering theory in the energy space for a class of nonlinear wave equations. Comm. Math. Phys. 123 (1989), 535-573.

[3] Glassey, R. T.: Finite-time blow-up for solutions of nonlinear wave equations. Math. Z. 177 (1981), 323-340.

[4] Glassey, R. T.: Existence in the large for $\square u=F(u)$ in two space dimensions. Math. Z. 178 (1981), 233-261.

[5] Harmse, J.: On Lebesgue space estimates for the wave equation. Indiana Univ. Math. J. 39 (1990), 229-248.

[6] Hidano, K.: Small data scattering and blow-up for a wave equation with a cubic convolution. Funkcial. Ekvac. 43 (2000), 559-588.

[7] Hidano, K.: Morawetz-Strichartz estimates for spherically symmetric solutions to wave equations and applications to semilinear Cauchy problems. Differential Integral Equations 20 (2007), 735-754.

[8] Hidano, K. And Kurokawa, Y.: Local existence of minimal-regularity radial solutions to semi-linear wave equations. Preprint.

[9] John, F.: Blow-up of solutions of nonlinear wave equations in three space dimensions. Manuscripta Math. 28 (1979), 235-268.

[10] Li, T. T. And Zhou, Y.: A note on the life-span of classical solutions to nonlinear wave equations in four space dimensions. Indiana Univ. Math. J. 44 (1995), 1207-1248. 
[11] Lindblad, H. And Sogge, C. D.: On existence and scattering with minimal regularity for semilinear wave equations. J. Funct. Anal. 130 (1995), 357-426.

[12] Lindblad, H. And Sogge, C. D.: Long-time existence for small amplitude semilinear wave equations. Amer. J. Math. 118 (1996), 1047-1135.

[13] Nakamura, M. and Ozawa, T.: The Cauchy problem for nonlinear wave equations in the homogeneous Sobolev space. Ann. Inst. H. Poincaré Phys. Théor. 71 (1999), 199-215.

[14] Pecher, H.: Nonlinear small data scattering for the wave and KleinGordon equation. Math. Z. 185 (1984), 261-270.

[15] Schaeffer, J.: The equation $u_{t t}-\Delta u=|u|^{p}$ for the critical value $p$. Proc. Roy. Soc. Edinburgh Sect. A 101 (1985), 31-44.

[16] Smith, H. F. And Sogge, C. D.: Global Strichartz estimates for nontrapping perturbations of the Laplacian. Comm. Partial Differential Equations 25 (2000), 2171-2183.

[17] Sogge, C. D.: Lectures on nonlinear wave equations. Monographs in Analysis, II. International Press, Boston, MA, 1995.

[18] Stein, E. M.: Harmonic Analysis: real-variable methods, orthogonality, and oscillatory integrals. Princeton Mathematical Series 43. Monographs in Harmonic Analysis, III. Princeton University Press, Princeton, NJ, 1993.

[19] Sugimoto, M.: Global smoothing properties of generalized Schrödinger equations. J. Anal. Math. 76 (1998), 191-204.

[20] TAO, T.: Spherically averaged endpoint Strichartz estimates for the twodimensional Schrödinger equation. Comm. Partial Differential Equations 25 (2000), 1471-1485.

Recibido: 7 de noviembre de 2006

Kunio Hidano

Department of Mathematics

Faculty of Education

Mie University

1577 Kurima-machiya-cho

Tsu, Mie Prefecture 514-8507, Japan

hidano@edu.mie-u.ac.jp

The author was supported in part by the Grant-in-Aid for Young Scientists (B) (No.18740069), The Ministry of Education, Culture, Sports, Science and Technology, Japan. 